\title{
Selecting Patients for Hyperthermia Treatment Based on Patient Backgrounds
}

\author{
NAOYUKI SAKAMOTO ${ }^{1}$, SATOSHI KOKURA ${ }^{2 *}$, TAKESHI ISHIKAWA ${ }^{2}$, \\ MARI TANIGAWA ${ }^{1}$, YUJI NAITO ${ }^{2}$, TOSHIKAZU YOSHIKAWA ${ }^{2}$
}

\author{
${ }^{1}$ Hyakumanben Clinic, 103-5 Tanaka Monzen-cho, Sakyo-ku, Kyoto 606-8255, Japan \\ ${ }^{2}$ Molecular Gastroenterology and Hepatology, Graduate School of Medical Sciences, Kyoto Prefectural University \\ of Medicine, 465 Kajii-cho, Kamigyo-ku, Kyoto 602-8566, Japan
}

\begin{abstract}
Use of hyperthermia as cancer treatment has often been impossible to continue at once following exacerbation of general condition due to cancer growth. However, no formal consensus has yet been defined regarding the acceptability of hyperthermia, so selection of patients for hyperthermia is not based on agreed clinical criteria. Aiming at the establishment of agreed clinical criteria concerning the selection of patients for hyperthermia, this report examined the treatment acceptability of hyperthermia based on patient backgrounds before advanced cancer treatment. Subjects comprised 45 patients with various advanced cancers treated with regional hyperthermia combined with chemo- or immunotherapy who visited our clinic between July 2008 and May 2009. Group A $(n=24)$ underwent hyperthermia $\geq 8$ times, while Group B $(\mathrm{n}=21)$ underwent hyperthermia $\leq 7$ times (mean, 4.19 times). We investigated pretreatment laboratory data, body mass index, performance status (PS), Glasgow prognostic score (GPS), and quality of life (QOL). Patients with poor scores for both PS and GPS dropped out early. In these patients, elevations in both lactate dehydrogenase (LDH) and C-reactive protein (CRP) levels tended to be present in addition to hypoalbuminemia. QOL in Group B was already impaired before initiation of hyperthermia. PS and GPS appear to represent the most important factors when judging the acceptability of hyperthermia, while LDH, CRP, and albumin levels may help such judgments. The acceptability of hyperthermia can be predicted using patient background as evidenced by laboratory data and general conditions, including QOL, before cancer treatment. The results justified further examination in a large number of patients to aim the establishment of agreed clinical criteria concerning the selection of patients for hyperthermia.
\end{abstract}

Key Words: acceptability, hyperthermia, performance status (PS), Glasgow prognostic score (GPS), quality of life (QOL)

Received 9 March, 2011, Accepted 2 June, 2011. *Corresponding author; Tel, +81-75-251-5519; Fax, +81-75-251-0710;

e-mail, s-kokura@koto.kpu-m.ac.jp

doi : $10.3191 /$ thermalmed.27.51

C 2011 Japanese Society for Thermal Medicine 


\section{Introduction}

Hyperthermia has gained attention as an anticancer therapy, and hyperthermia combined with chemo- and radiotherapy has reportedly proven advantageous ${ }^{1-4)}$. Hyperthermia is mostly used in patients with advanced cancer. Some patients for whom hyperthermia is planned have already undergone cancer therapies and might be refractory to these treatments. The general condition in such patients is likely to be poor. Conversely, hyperthermia can be performed with few side effects compared with other anticancer modalities, and is thus readily performed. In many cases, however, hyperthermia cannot be continued because of deterioration of the general condition due to cancer growth. In such cases, evaluation of the patient suitability to undergo hyperthermia has clearly been inadequate. In Response Evaluation Criteria In Solid Tumors (RECIST) Guidelines, it was reported that in the context of phase II studies where the beneficial effect of therapy is not known, follow-up of every other cycle (i.e., 6-8 weeks) seems a reasonable norm ${ }^{5}$. Considering that hyperthermia must be used consistently for a certain period to obtain effects, the ability of the patient to accept hyperthermia for this duration needs to be assessed. Without sufficient evaluation of acceptability before treatment, a scientific basis for considering that hyperthermia should be performed is lacking. The purpose of this study was to examine treatment acceptability of hyperthermia based on patient backgrounds, and to determine factors predicting treatment acceptability.

\section{Material and methods}

\section{Patient characteristics}

Subjects comprised 45 patients with various advanced cancers who were treated with regional hyperthermia combined with chemo- or immunotherapy who visited our clinic between July 2008 and May 2009. We wanted to aim to evaluate the treatment acceptability of hyperthermia not based on the cancer type, the stage of disease, but only based on patient backgrounds. So, we intentionally considered that the clinical profile and demographics vary widely. Unresectable local advanced cancer was detected in 36 out of 45 cases. Unresectable recurrent cancer was detected in 9 out of 45 cases. Tumor origin of patient was various in this study - esophagus, stomach, colon, small intestine, pancreas, liver, biliary tract, lung breast, ovary, uterus, and other for example. Clinical characteristics of patients are summarized in Table I. By the way, in this study, aiming the examination for the treatment acceptability

Table I. Clinical characteristics

\begin{tabular}{|c|c|c|}
\hline & & Group A / Group B \\
\hline & Age: median (range) & $60.1(37-72) / 66.0(49-79)$ \\
\hline & Male / Female & $(13 / 11) /(15 / 6)$ \\
\hline$(\mathrm{Ad}$ & $\begin{array}{l}\text { Clinical stage } \\
\text { d-inoperable / unresectable-recurrent) }\end{array}$ & $(18 / 6) /(18 / 3)$ \\
\hline \multirow[t]{2}{*}{ Tumor origin } & $\begin{array}{l}\text { esophagus, stomach, colon, small intestine } \\
\text { pancreas, liver, biliary tract } \\
\text { lung } \\
\text { breast, ovary, uterus } \\
\text { other }\end{array}$ & $\begin{array}{l}8 / 4 \\
8 / 12 \\
3 / 3 \\
4 / 1 \\
1 / 1\end{array}$ \\
\hline & $\operatorname{PS}(0 / 1 / 2 / 3 / 4)$ & $(17 / 7 / 0 / 0 / 0) /(2 / 10 / 7 / 1 / 0)$ \\
\hline
\end{tabular}


of hyperthermia, we excluded the patient combined the concurrent chemo- or immunotherapy that have brought the serious adverse events when hyperthermia was continued. Subjects didn't comprise patients who resulted in dropping out hyperthermia due to impossibility of taking a load by hyperthermia.

\section{Hyperthermia}

Hyperthermia was applied using a Thermotron RF-8 heating device (Yamamoto Vinita, Osaka, Japan). Heating time for hyperthermia was $40 \mathrm{~min}$, with hyperthermia was performed once a week. In this study, both the upper and lower electrodes were $30 \mathrm{~cm}$ in diameter and placed on opposite sides of the abdominal, pelvic or thoracic region. Treatment posture was the supine or prone position. The temperature of tumor during hyperthermia was not measured. Increased until reaching the patient's tolerance threshold, the power range was adjusted from 600 to $1500 \mathrm{~W}$ according to feedback from the patient's complaint. During hyperthermia, the patient's skin was cooled with a circulating water system.

\section{Combination therapy}

Most patients received hyperthermia in combination with chemo- or immunotherapy. Chemotherapy followed appropriate protocols based on tumor origins. In this study, the aim was to examine the treatment acceptability of hyperthermia. So, we enrolled only the patients for whom chemotherapeutic agents were not influenced to continue hyperthermia. Most patients have been treated by standard chemotherapeutic agent, such as 5-fluorouracil, S-1, gemcitabine, irinotecan, oxaliplatin, docetaxel, paclitaxel, and cisplatin. As immunotherapy, adoptive cell therapy or autologous cancer vaccine was applied. These immunotherapies have been approved by the ethics committee of our institution.

\section{Blood parameters}

Routine blood examinations including hematology, blood biochemistry and serology were undertaken before initiating hyperthermia. By the way, in this study, we have examined only the patient's data of which every change of the parameter was influenced by cancer growth rather than the other cause such as the inflammation not based on cancer growth, the infection, the organ failure.

\section{Examination of general condition}

To examine the general condition of each patient before hyperthermia, body mass index and performance status (PS) according to Eastern Cooperative Oncology Group criteria were surveyed. Furthermore, as a prognostic score reflecting general condition, the Glasgow Prognostic Score (GPS) based on C-reactive protein (CRP) and albumin

Table II. Glasgow prognostic score (GPS)

levels was applied. The GPS was surveyed as previously described ${ }^{6,7)}$. A summary of how to apply the GPS is shown in Table II.

\begin{tabular}{ll}
\hline 1) $\mathrm{CRP}>1 \mathrm{mg} / \mathrm{dl}$ & \\
2) $\mathrm{Alb}<3.5 \mathrm{~g} / \mathrm{dl}$ & score \\
& $\rightarrow 2$ \\
1) and 2) & $\rightarrow 1$ \\
1) or 2) & $\rightarrow 0$ \\
Neither 1) or 2) &
\end{tabular}




\section{Assessment of quality of life (QOL)}

To interview and assess QOL in patients, the EORTC QLQ-C30 ${ }^{8}$ (version 3.0) questionnaire was used. This questionnaire has recently been used to evaluate clinical benefits induced by cancer therapy ${ }^{9,10)}$. QOL was determined before and after hyperthermia, which was performed for 8 weeks.

\section{Statistical analysis}

Variables were compared by means of a non-parametric test (Mann-Whitney) for intergroup comparisons. A paired t-test was used to compare results before and after treatment. Values of $p<$ 0.05 were considered statistically significant.

\section{Results}

\section{Acceptable rate}

In this study, we led the patient to dropout hyperthermia if the medical specialists judged that they couldn't treat the patient by hyperthermia due to the exacerbation of general condition affected only by cancer growth, not by the adverse event of chemo- or immunotherapy, or hyperthermia. Response Evaluation Criteria In Solid Tumors (RECIST) Guidelines is used worldwide as agreed clinical criteria. In this guideline, it is described clearly that follow-up of a cancer treatment usually seems to be checked up every $6-8$ weeks $^{5}$. Considering this definition of follow-up, the patient who received weekly hyperthermia $\geq 6-8$ times only have to be judged as acceptable patient, because we thought hyperthermia must be administered consistently for a certain period both to obtain effects and to evaluate effects. According to the NCI Common Terminology Criteria for Adverse Events (CTCAE), we evaluate the scale for adverse events with which hyperthermia or other cancer treatment combined with hyperthermia have related. As a result, no patients have experienced treatment-related adverse events Grade $\geq 3$ adverse events in this study. So, we judged that Hyperthermia was ended because of exacerbation of general condition rather than side effects of cancer therapies in this study. Actually, most patients who received hyperthermia only $\leq 7$ times have been impossible to continue at once following exacerbation of general condition due to cancer growth. So we defined that patients who could receive hyperthermia $\geq 8$ times were suitable. A total of 24 patients (53.3\%; Group A) received hyperthermia $\geq 8$ times. The remaining 21 patients (46.7\%; Group B) were treated using hyperthermia $\leq 7$ times. The mean number of hyperthermia treatments in Group B was thus 4.19 , so hyperthermia seemed largely unacceptable for Group B. In Group B, 4 patients (19.0\%) have pointed out both peritoneal dissemination and liver metastasis. 4 patients (19.0\%) were accompanied with pleuritis carcinomatosa.

\section{Blood parameters}

Lactate dehydrogenase (LDH) and CRP levels were significantly lower in Group A than in Group $B(p=0.022$ and $p<0.0001$, respectively) (Fig. 1a, b). Albumin level was significantly higher in Group A than in Group B $(p=0.0005)$ (Fig. 1c). 
a)

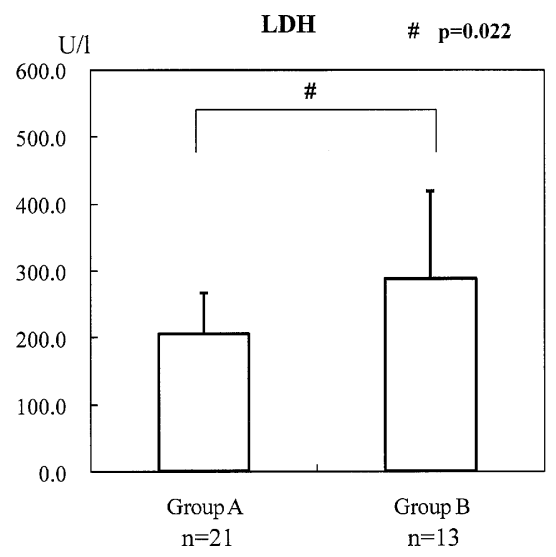

c)

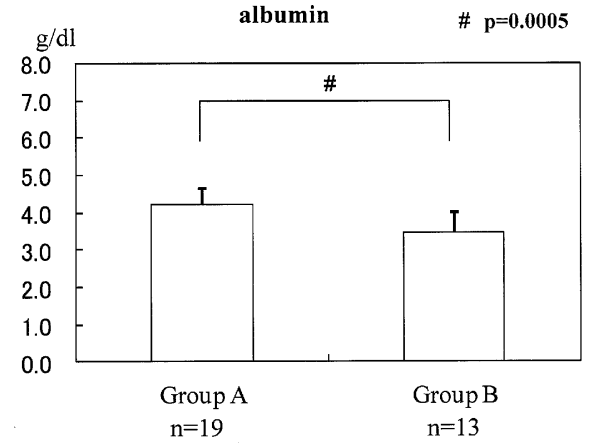

b)

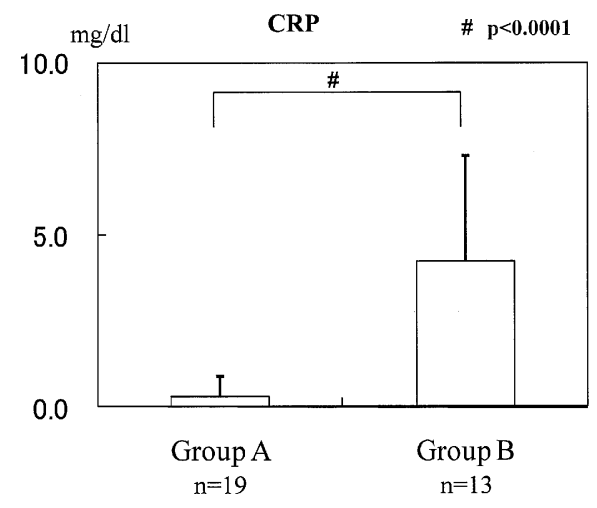

Fig. 1. LDH, CRP, and albumin levels differed significantly between Groups A and B. a, b) LDH and CRP levels were significantly elevated in Group B compared with Group A $(\mathrm{p}=0.022$ and $\mathrm{p}<0.0001$, respectively). c) Albumin level was significantly decreased in Group B $(\mathrm{p}=0.0005)$. Values are expressed as the mean \pm SD.

\section{General conditions}

PS was higher in Group A than in Group B $(\mathrm{p}<0.0001)$. GPS was lower in Group A than in Group B $(p=0.0027)$. General condition was thus significantly better in Group A than in Group B (Fig. 2a, b). Furthermore, patients with both high PS and GPS tended to drop out in the early stages.

a)

$$
\text { Performance status ( PS ) }
$$

\# $\quad \mathbf{p}<0.0001$

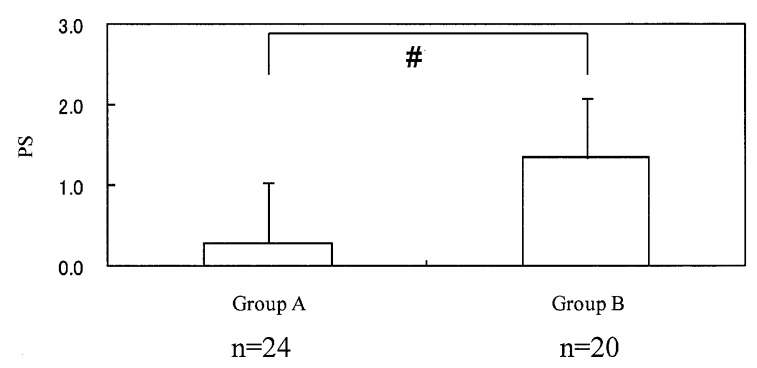

b)

Glasgow Prognostic score $<$ GPS $>$

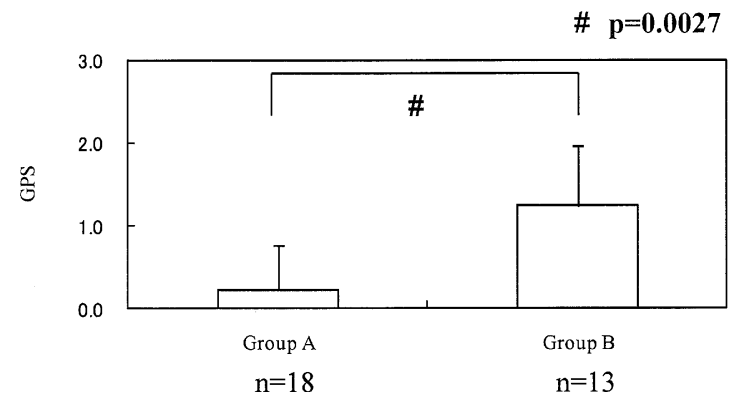

Fig. 2. General condition in Group B according to PS and GPS was significantly worse in Group B than in Group A. a) PS was worse in Group B than in Group A ( $<<0.0001)$. b) GPS was higher in Group B than in Group A $(p=0.0027)$. Values are expressed as the mean \pm SD. 
a)

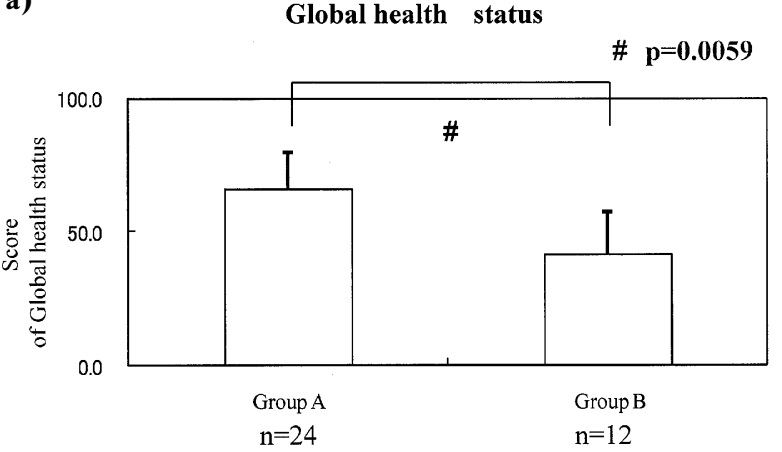

b)

The average of functional scales

\# $\mathbf{p}=\mathbf{0 . 0 0 2 8}$

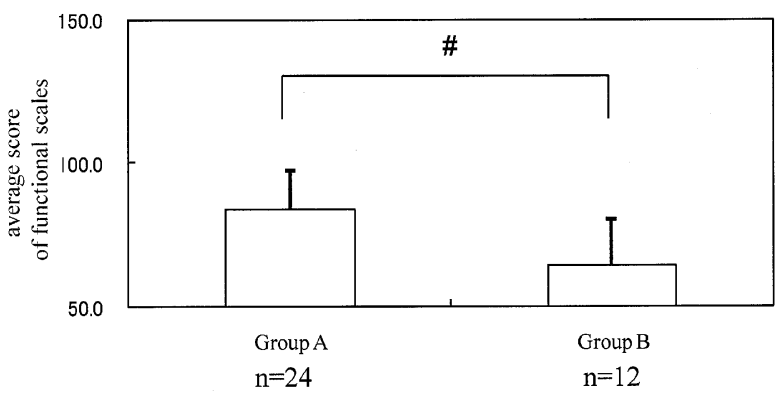

Fig. 3. QOL was already low in terms of global health status and mean scores for functioning scales in Group B before hyperthermia $(p=0.0059$ and $p=0.0028$, respectively). Values are expressed as the mean $\pm S D$. To assess global health status and mean scores for functioning scales, the EORTC QLQ-C30 ${ }^{8)}$ (version 3.0) questionnaire was used.

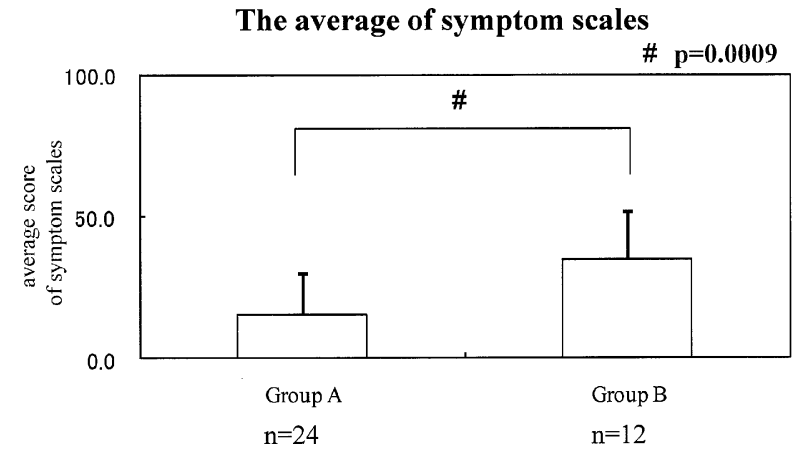

a)

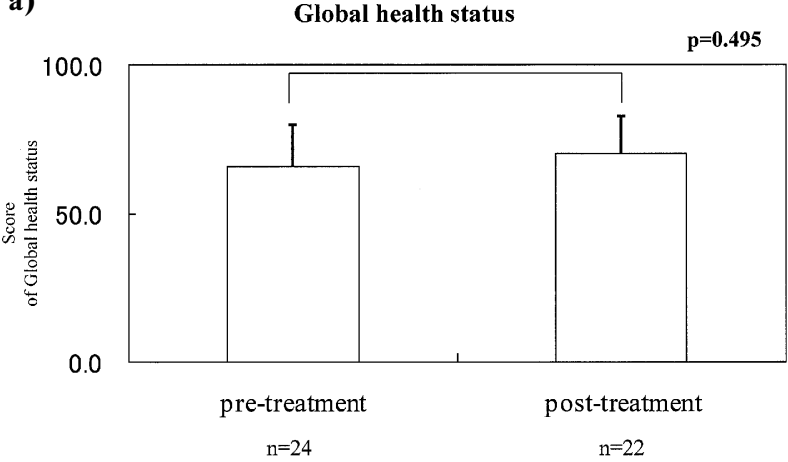

c)

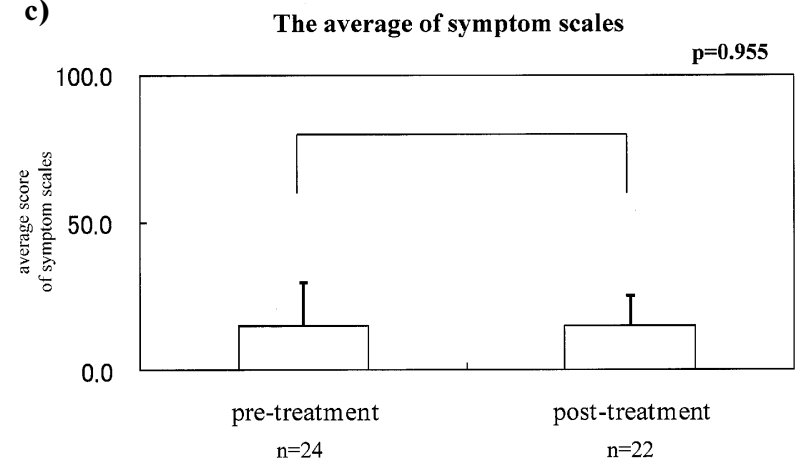

Fig. 4. Moderate and severe symptoms had already appeared in Group B before hyperthermia ( $\mathrm{p}=$ 0.0009 ). Values are expressed as the mean $\pm S D$. To assess mean scores for symptom scales, the EORTC QLQ-C30 ${ }^{8}$ (version 3.0) questionnaire was used.

b)

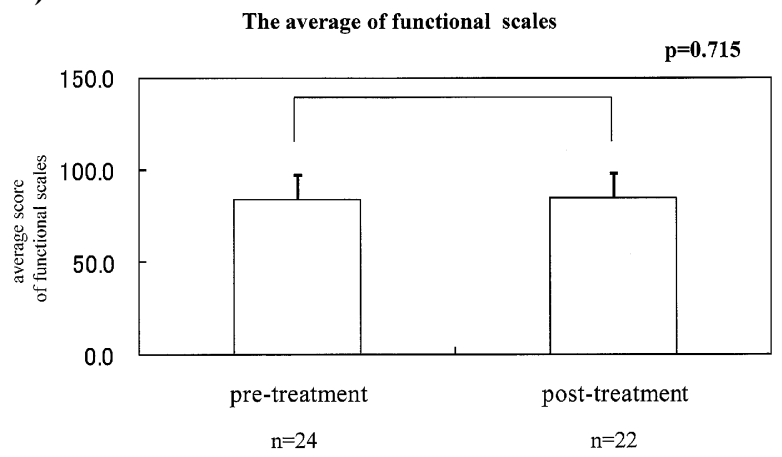

Fig. 5. a-c) QOL in Group A was examined again after performing hyperthermia 8 times. QOL in Group A was unimpaired compared with that before hyperthermia. Values are expressed as the mean \pm SD. To assess global health status, mean scores for functioning scales, and mean scores for symptom scales the EORTC QLQ-C308) (version 3.0) questionnaire was used. 
$Q O L$

QOL was already worse in terms of global health status and mean scores for functioning scales in Group B than in Group A before hyperthermia ( $p=0.0059$ and $p=0.0028$, respectively) (Fig. 3a, b). Some moderate or severe symptoms had already appeared in Group B before hyperthermia $(p=0.0009)$ (Fig. 4). QOL in Group A was observed again after performing hyperthermia 8 times. QOL in Group A was not impaired compared with that before hyperthermia (Fig. 5a-c).

\section{Discussion}

Hyperthermia is mostly used in patients with advanced cancer. Occasionally, eligible patients have already been treated by various cancer therapies, and might already be refractory to them. The ratio of patients with highly progressive cancer is thus considerably high at the start of hyperthermia. For patients with progressive cancer, early cancer progression after hyperthermia leads to deterioration of general condition and often results in discontinuation of hyperthermia. Hyperthermia is not invasive and can be performed with few side effects compared with other anticancer modalities, and so is easily performed. According to these backgrounds, insufficient evaluation of acceptability leads to discontinuation of hyperthermia in the early stage. Response Evaluation Criteria In Solid Tumors (RECIST) Guidelines is used worldwide as agreed clinical criteria. In this guideline, it is described clearly that follow-up of a cancer treatment usually seems to be checked up every $6-8$ weeks $^{5}$. This definition of follow-up have suggested us that the patient who received weekly hyperthermia $\geq 6-8$ times only should be judged to be acceptable because we thought hyperthermia must be administered consistently for a certain period both to obtain effects and to evaluate effects. In this study, the ratio of dropout patients who underwent hyperthermia $\leq 7$ times was high, at $47 \%$. In such cases, evaluation of the patient suitability to undergo hyperthermia has clearly been inadequate. We thus think that sufficient evaluation of acceptability would decrease the number of patients who start hyperthermia but dropout in the early stages. Especially, we wanted to aim to plan agree clinical criteria concerning treatment acceptability of hyperthermia not based on the cancer type, the stage of disease, but only based on patient backgrounds, such as Palliative Prognostic Index ${ }^{11)}$ that defined about patient's prognosis. So, we intentionally considered that the clinical profile and demographics vary widely. Generally, the general condition of the cancer-bearing patient changes depending on the cancer type and the stage of disease. However, when cancer progresses to some degree, the general condition and QOL change regardless of the cancer type. To evaluate the acceptability of hyperthermia regardless of the cancer type, we try to analyze various parameters.

We first examined blood parameters to evaluate the acceptability of hyperthermia. In patients who dropped out early (Group B), LDH and CRP were significantly elevated and albumin was significantly decreased compared with patients who could continue hyperthermia (Group A). LDH, CRP, and albumin levels were thus considered as factors reflecting cancer progression to some degree, as reported elsewhere ${ }^{12-14)}$. These values may thus represent predictive factors for the acceptability of hyperthermia.

To evaluate general condition before the onset of hyperthermia, BMI, PS, and GPS were examined. No significant differences in BMI were seen between Groups A and B. Significantly, PS was poorer in Group B than in Group A, and GPS was higher in Group B than in Group A. Considering this result, 
general condition in Group B was worse than in Group A. Patients with $\mathrm{PS} \geq 2$ and GPS $\geq 1$ almost always dropped out (Table III). Patients with not only both peritoneal dissemination and liver metastasis, but also with pleuritis carcinomatosa showed a tendency to drop out in the early stages. GPS in most of these patients was

Table III. Distribution of PS and GPS

\begin{tabular}{|l|l|r|r|}
\hline PS4 & & & \\
\hline PS3 & & & $0 \%: 7.7 \%$ \\
\hline PS2 & & $0 \%: 23.1 \%$ & $0 \%: 15.4 \%$ \\
\hline PS1 & $16.7 \%: 15.4 \%$ & $0 \%: 15.4 \%$ \\
\hline PS0 & $66.7 \%: 0 \%$ & $11.1 \%: 7.7 \%$ & \\
\hline & GPS0 & GPS1 & GPS2 \\
\hline
\end{tabular}

2.

In the evaluation of QOL, global health status and mean scores for functional scales were decreased and mean scores for symptom scales were increased in patients who dropped out early. These patients had probably already complained of moderate or severe symptoms. QOL in Group A was observed again after performing hyperthermia 8 times. QOL in Group A was not impaired compared with that before hyperthermia (Fig. 5a-c). This indicated that patients for whom QOL was unimpaired tended to be acceptable to continue hyperthermia treatment combined with chemo- or immunotherapy. Administration of this QOL questionnaire before implementing hyperthermia might thus be used as a prognostic factor.

Some reports have shown that eligible patients were feasible in some phase II studies concerning regional hyperthermia combined with chemotherapy ${ }^{15,16)}$. Selection of eligible patient have usually based on the information concerning ECOG performance status $\leq 2$, normal hematological index, acceptable biochemical analysis result (normal bilirubin level, alanine aminotransferase $2.5^{*}$ upper limit of normal (ULN) and creatinine level $\leq 1.5 \mathrm{ULN})$. Patients with metal foreign matter, fever $\left(\geq 38^{\circ} \mathrm{C}\right)$, significant hemorrhagic tendency, and seriously impaired function of lung or heart were excluded. These baselines select the patients who can receive the hyperthermia at least once. However, only by usual eligibility criteria, we can't select those patients who can be administered the hyperthermia consistently for a certain period to obtain the effect. On the other hand, no previous study has shown detail criteria about the treatment acceptability of hyperthermia based on patient backgrounds reflected both general condition and QOL correlated with cancer growth. Based on our examination, the acceptability of hyperthermia seems to be predictable using both PS and GPS as the main evaluation items, and QOL, LDH, CRP, and albumin as supplementary evaluation items. Characteristics of patients relevant for evaluating and predicting the acceptability of hyperthermia are summarized in Table IV. Using these factors, inappropriate initiation of hyperthermia might be decreased. Sufficient evaluation of acceptability using routine blood examination, objective

Table IV. Prediction of acceptability

\begin{tabular}{|c|c|c|c|}
\hline PS4 & & & \\
\hline PS3 & & & \\
\hline PS2 & & & \\
\hline PS1 & & & \\
\hline PSO & & & \\
\hline & GPSO & GPS1 & GPS2 \\
\hline & & \multicolumn{2}{|c|}{$\begin{array}{l}\text { maybe acceptable } \\
\text { borderline }\end{array}$} \\
\hline
\end{tabular}


judgment of PS and a QOL questionnaire may allow appropriate selection of patients for hyperthermia. Further clinical study for examinations about the treatment acceptability of hyperthermia in the patients with various cancers is recommended.

\section{References}

1) Huilgol N.G., Gupta S., Dixit R. : Chemoradiation with hyperthermia in the treatment of head and neck cancer. Int J Hyperthermia, $26: 21-25,2010$.

2) Oldenborg S., Van Os R.M., Van rij C.M., Crezee J., Van de Kamer J.B., Rutgers E.J., Geijsen E.D., Zum vörde sive vörding P.J., Koning C.C., Van tienhoven G.: Elective re-irradiation and hyperthermia following resection of persistent locoregional recurrent breast cancer : A retrospective study. Int J Hyperthermia, 26 : 136-144, 2010.

3) Fotopoulou C., Hee Cho C., Kraetschell R., Gellermann J., Wust P., Lichtenegger W., Sehouli J. : Regional abdominal hyperthermia combined with systemic chemotherapy for the treatment of patients with ovarian cancer relapse : Results of a pilot study. Int J Hyperthermia, 26: 118-126, 2010.

4) Ishikawa T., Kokura S., Inui T., Suzuki K., Matsuyama R., Okita M., Isozaki Y., Nagao Y., Ando T., Naito Y., Yoshida N., Yoshikawa T. : Effects of sequential combination of hyperthermia and gemcitabine in the treatment of advanced unresectable pancreatic cancer : A retrospective study. Thermal Med, 24 : 131-139, 2008.

5) Eisenhauer E.A., Therasse P., Bogaerts J., Schwartz L.H., Sargent D., Ford R., Dancey J., Arbuck S., Gwyther S., Mooney M., Rubinstein L., Shankar L., Dodd L., Kaplan R., Lacombe D., Verweij J. : New response evaluation criteria in solid tumours: Revised RECIST guideline (version 1.1). Eur J Cancer, 45 : 228-247, 2009.

6) Forrest L.M., McMillan D.C., McArdle C.S., Angerson W.J., Dunlop D.J. : Evaluation of cumulative prognostic scores based on the systemic inflammatory response in patients with inoperable non-small-cell lung cancer. Br $\mathrm{J}$ Cancer, 89 : 1028-1030, 2003.

7) Forrest L.M., McMillan D.C., McArdle C.S., Angerson W.J., Dunlop D.J. : Comparison of an inflammation-based prognostic score (GPS) with performance status (ECOG) in patients receiving platinum-based chemotherapy for inoperable non-small-cell lung cancer. Br J Cancer, 90 : 1704-1706, 2004.

8) Aaronson N.K., Ahmedzai S., Bergman B., Bullinger M., Cull A., Duez N.J., Filiberti A., Flechtner H., Fleishman S.B., de Haes J.C., Kaasa S., Klee M., Osoba D., Razavi D., Rofe P.B., Schraub S., Sneeuw K., Sullivan M., Takeda F. : The European Organization for Research and Treatment of Cancer QLQ-C30: A quality-of-life instrument for use in international clinical trials in oncology. J Natl Cancer Inst, 85 : 365-376, 1993.

9) Homs M.Y., Essink-Bot M.L., Borsboom G.J., Steyerberg E.W., Siersema P.D., Dutch SIREC Study Group : Quality of life after palliative treatment for oesophageal carcinoma - a prospective comparison between stent placement and single dose brachytherapy. Eur J Cancer, 40 : 1862-1871, 2004.

10) Schulte T., Schniewind B., Walter J., Dohrmann P., Küchler T., Kurdow R. : Age-related impairment of quality of life after lung resection for non-small cell lung cancer. Lung Cancer, 68 : 115-120, 2010.

11) Morita T., Chinone Y., Ikenaga M., Miyoshi M., Nakaho T., Nishitateno K., Sakonji M., Shima Y., Suenaga K., Takigawa C., Kohara H., Tani K., Kawamura Y., Matsubara T., Watanabe A., Yagi Y., Sasaki T., Higuchi A., Kimura H., Abo H., Ozawa T., Kizawa Y., Uchitomi Y.: Japan Pain, Palliative Medicine, Rehabilitation, and Psycho-Oncology Study Group. Ethical validity of palliative sedation therapy: A multicenter, prospective, observational study conducted on specialized palliative care units in Japan. J Pain Symptom Manage, 30: 308-319, 2005.

12) Koch A., Fohlin H., Sörenson S. : Prognostic significance of C-reactive protein and smoking in patients with advanced non-small cell lung cancer treated with first-line palliative chemotherapy. J Thorac Oncol, 4: 326-332, 2009.

13) Brown D.J., Milroy R., Preston T., McMillan D.C. : The relationship between an inflammation-based prognostic score 
Thermal Med, $27[2]:$ 51-60, 2011.

(Glasgow Prognostic Score) and changes in serum biochemical variables in patients with advanced lung and gastrointestinal cancer. J Clin Pathol, 60 : 705-708, 2007.

14) Tas F., Duranyildiz D., Oguz H., Camlica H., Yasasever V., Topuz E. : Serum vascular endothelial growth factor (VEGF) and bcl-2 levels in advanced stage non-small cell lung cancer. Cancer Invest, 24 : 576-580, 2006.

15) Shen H., Li X.D., Wu C.P., Yin Y.M., Wang R.S., Shu Y.Q. : The regimen of gemcitabine and cisplatin combined with radio frequency hyperthermia for advanced non-small cell lung cancer: A phase II study. Int J Hyperthermia, 27 : 27-32, 2011.

16) Jiang Z., Yan W., Ming J., Yu Y.: Docetaxel weekly regimen in conjunction with RF hyperthermia for pretreated locally advanced non-small cell lung cancer: A preliminary study. BMC Cancer, 7 : 189, 2007. 